\title{
Urologie umfasst mehr
}

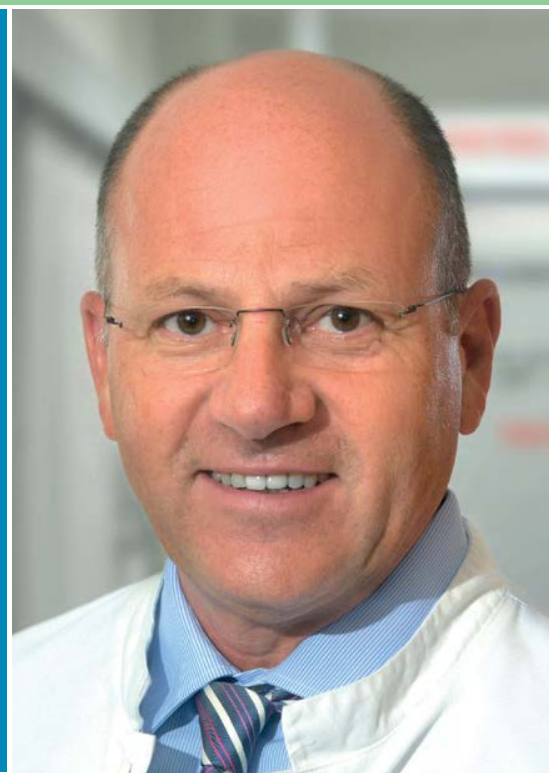

Liebe Leserin, lieber Leser,

das Motto „Urologie umfasst mehr“ steht für die Vielfalt unseres Fachs über geschlechts- und altersspezifische Grenzen hinaus. Denn der Urologe und die Urologin sind mehr als „der Arzt für den Mann und die Prostata"! Die Betonung dieses männerärztlichen Fokus hat fast unbemerkt dazu geführt, dass der immanente urologische Therapieauftrag, die Behandlung von Nieren-, Blasen- und Genitalerkrankungen beider Geschlechter und aller Altersgruppen, in der medialen Berichterstattung und der Wahrnehmung der Öffentlichkeit in den Hintergrund getreten ist. Selbstverständlich muss der urologisch erkrankte Mann behandelt werden - aber auch die urologisch erkrankte Frau und das urologisch erkrankte Kind. Deshalb müssen wir in der Außendarstellung unsere organbezogenen Diagnose- und Therapiekompetenzen intensiver strategisch verfolgen und gezielter dafür werben, dass in der Urologie nicht nur Männer, sondern auch Frauen und Kinder behandelt werden.

Die Beiträge dieses Kongresshefts sollen etwas von der Vielfalt unseres Fachs wiedergeben. Der Beitrag von Priv.-Doz. Dr.
Andreas Wiedemann „Kleinkapazitäre Blase: Medikamente und/oder Miktionstraining?" beschäftigt sich mit der Frage, welche Evidenz der verhaltenstherapeutischen Maßnahme eines Blasentrainings zugrunde liegt. Dieser Beitrag ergänzt die diesjährige Initiative der Deutschen Gesellschaft für Urologie, den urogynäkologischen Aspekt unseres Fachs zu stärken. Deshalb haben wir ein Ausbildungsmodul „Assistenten für Urologische Kontinenztherapie“ erarbeitet. Dieses für medizinische Fachangestellte und urologisch Pflegende geschaffene Angebot wird in Kooperation mit dem BDU ab 2016 über die Akademie der Deutschen Urologen angeboten. Dadurch sollen flächendeckend und mit unkompliziertem Zugang Ansprechpartner in urologischen Praxen und Kliniken ausgebildet werden, sodass Betroffenen mit Blasenfunktionsstörung neben der ärztlichen Beurteilung eine zusätzliche qualifizierte und adäquate Diagnostik sowie Verlaufskontrolle ermöglicht werden kann.

In diesem Zusammenhang ist auch der Beitrag von Dr. Alfons Gunnemann aus Detmold zu sehen, der sich in dem Artikel „Die Harnröhrenenge und Blasenhalsstenose der Frau - Fakt oder Mythos -
Was ist zu tun?" mit einem der am wenigsten beachteten Organe der Frau, der Urethra und dem Blasenhals, beschäftigt. Unter anderem wird eine Übersichtsarbeit von Osman et al. vorgestellt, die in einer systematischen Literaturrecherche herausgefunden haben, dass alle publizierten Daten zur Harnröhrenstriktur der Frau lediglich 221 auswertbare Patientinnen aufweisen und zudem alle Daten retrospektiv erhoben wurden. Ähnliches gilt für die Entität der Blasenhalsstenose bei der Frau. Die Übersichtsarbeit zeigt in schon beinahe erschreckender Weise, wie wenig evidenzbasierte Erkenntnisse über Funktionsstörungen und Erkrankungen dieses Organs bei der Frau vorliegen.

Insbesondere in der ambulanten Urologie ist der chronische Beckenbodenschmerz ein häufiger, aber bezüglich der diagnostischen Eindeutig- und Therapierbarkeit allerdings auch frustrierender Befund. Dr. Oliver Moormann gibt in seiner Arbeit „Chronischer Beckenbodenschmerz Alternative Behandlungsverfahren“ einen Überblick über die verschiedenen Therapievarianten. Da die Effekte der konventionellen urologischen Therapie beim chronischen Beckenschmerzsyndrom (CPPS=Chronic Pelvic Pain Syndrom) häu- $\longmapsto$ Außerdem in diesem Heft

\section{Klinik und Praxis}

$\checkmark$

„Doktor, was kann ich selber tun?“ - Wie Sie Krebspatienten im Hinblick auf Komplementärmedizin beraten können, lesen Sie ab ๑ Seite 363.

\section{Referiert und kommentiert}

$\nabla$

MS Fazeli und Kollegen haben in einer Metaanalyse die Studienergebnisse zur Biofeedbacktherapie bei Kindern zusammengetragen und bewertet. Wir haben die Analyse auf $\bullet$ Seite 345 für Sie zusammengefasst.

\section{Recht in der Praxis}

$\nabla$

Das Bundesministerium für Gesundheit möchte Anwendungen der elektronischen Gesundheitskarte mithilfe des E-HealthGesetzes beschleunigen ( $\odot$ Seite $\mathbf{3 6 6}$ ). 
fig unzureichend sind und meist mehr als ein Organ betroffen ist, wird eine multimodale und interdisziplinäre Therapie empfohlen.

Die Steintherapie ist unverändert eine auch quantitativ herausragende urologische Erkrankung. Ein häufiges Problem ist dabei die Frage, ob und wann asymptomatische Nierensteine behandelt werden müssen. In der Übersichtsarbeit von Dr. Andreas Neisius aus Mainz „Asymptomatische Nierensteine: Wann Active surveillance, wann Therapie?" werden die verfügbaren Studien analysiert und der Autor kommt zu der praktisch relevanten Schlussfolgerung, dass bei asymptomatischen und nicht obstruierenden Nierensteinen bis zu einer Steingröße von $10 \mathrm{~mm}$ ein Zuwarten mit geringem Risiko gerechtfertigt erscheint, während man bei Steinen mit einem deutlichen Steinwachstum eher zu einer Intervention raten sollte.

Ein wichtiges Thema des diesjährigen Urologischen Kongresses ist die Kinderurologie. Es zeigt sich zunehmend, dass wir Urologen aufpassen müssen, dass häufige kinderurologische Erkrankungen aus rein strategisch-ökonomischen Gründen nicht der Urologie weggenommen und den Kinderchirurgen zugewiesen werden, da andernfalls all die kinderurologischen Abteilungen nicht existenzfähig sind. In der Zukunft sollte die Deutsche Gesellschaft für Urologie noch mehr wissenschaftliche Aktivitäten in der Kinderurologie fördern, um den „Andrologieeffekt“ zu wiederholen. Bei der Andrologie hat sich nämlich gezeigt, dass es durch wissenschaftliche Aktivitäten vornehmlich in den Kliniken von Herrn Prof. Weidner und von Frau Prof. Kliesch gelungen ist, dieses Teilgebiet wieder natürlich in die Urologie zu reintegrieren. Der Beitrag der Pädiater Dr. Kai Hensel und Prof. Stefan Wirth aus Wuppertal „Aktuelle Defizite in der Therapie des Maldeszensus testis - Analyse eines Pädiaters“ zeigt, dass bei einem Maldeszensus testis die Rate der leitliniengerecht durchgeführten Orchidopexien mit Abschluss des ersten Lebensjahres mit 5\% (eigene Untersuchung der Autoren bei 3587 Fällen) immer noch erschreckend niedrig ist.

In diesem Zusammenhang möchte ich auf eine Initiative hinweisen, die die Deutsche Gesellschaft für Urologie deshalb dieses
Jahr durchgeführt hat. Wir haben begonnen, mit gezielten Aktivitäten in sozialen Netzwerken (Google, Facebook) vornehmlich junge und internetaffine Eltern über die häufigen kinderurologischen Erkrankungen (und uns Urologen als erste Ansprechpartner!) zu informieren. Diese aktuellen Daten zeigen, dass diese Informationsstrategie eine hohe Akzeptanz gefunden hat und sollte in den kommenden Jahren weiter verfolgt werden.

Ich hoffe, dass Ihnen die Lektüre des Kongresshefts spannende Anregungen und Informationen vermittelt und danke den Autoren für Ihre Beiträge.

Prof. Dr. Stephan Roth Präsident der Deutschen Gesellschaft für

Urologie 2014 / 2015

Direktor der Urologischen Klinik, HELIOS

Klinikum Wuppertal

Lehrstuhl für Urologie der Universität Witten-Herdecke

Heusnerstr. 40

42283 Wuppertal

Tel.: +49/202/896-3407

stephan.roth@helios-kliniken.de

\section{$\gg$ Außerdem in diesem Heft}

\section{Tipps und Tricks}

$\nabla$

Eingriffe im Unterbauch sind bei adipösen Patienten oft schwierig. Einen Tipp hierzu finden Sie auf $\bullet$ Seite 358.

\section{Operative Techniken}

$\nabla$

Drei Fortbildungspunkte können Sie in dieser Ausgabe sammeln, wenn Sie die CME-Fragen zum Thema spannungsfreie Schlingenoperationen richtig beantworten (o Seite 407).

\section{Risiken und Komplikationen}

$\nabla$

Welche Komplikationen beim Einlegen eines spannungsfreien Vaginalbands auftreten können, lesen Sie ab o Seite 410. 